\title{
Characterization of Wavelength Tunable Lasers for Future Optical Communication Systems
}

\author{
Prince M. Anandarajah \\ Research Institute for Networks and Communications Engineering, Dublin City University, Dublin 9, Ireland \\ Email: prince.anandarajah@dcu.ie \\ Aleksandra Kaszubowska-Anandarajah, Robert Maher, Kai Shi and Liam P. Barry \\ Research Institute for Networks and Communications Engineering, Dublin City University, Dublin 9, Ireland \\ Email: \{kaszubow, robert.maher, kaishi, barryl\}@eeng.dcu.ie
}

\begin{abstract}
The use of tunable lasers (TL) in dense wavelength division multiplexed (DWDM) networks for optical switching, routing and networking has gained a lot of interest in recent years. Employment of such TLs as tunable transmitters in wavelength packet switched (WPS) networks is one of the possible applications of these devices. In such systems, the information to be transmitted could be encoded onto a destination dependent wavelength and the routing of traffic could be performed on a packet-by-packet basis. The authors investigate the possibility of using TLs in DWDM WPS networks by focusing on the characterisation of the instantaneous frequency drift of a TL due to wavelength tuning and direct modulation. Characterization of the linewidth of the TLS is also presented to verify the feasibility of using TLs in systems employing advanced modulation formats.
\end{abstract}

Index Terms - tunable lasers, dense wavelength division multiplexing, frequency drift, direct modulation, optical communications

\section{INTRODUCTION}

The use of widely tunable lasers in Dense Wavelength Division Multiplexed (DWDM) systems, packet switched schemes and access networks, has gained increased interest in recent years. They are being introduced as alternatives to fixed wavelength sources to reduce inventory and to provide a more dynamic device for external or direct modulation [1]. In the above mentioned systems, the information to be transmitted could be encoded onto a destination dependant wavelength, generated by the tunable laser. This would allow for dynamic bandwidth provisioning and thus increasing the flexibility of the system. There are several different types of tunable lasers currently vying for supremacy in this market such as External Cavity Lasers (ECL), Vertical Cavity Lasers (VCSEL), Grating assisted co-directional Coupler with rear Sampled Reflector (GCSR) laser and the Sampled Grating Distributed Bragg Reflector (SGDBR) laser [2]. A major drawback associated with the external cavity laser is the complex manufacturing process that inevitably leads to high production costs, while the VCSEL and GCSR lasers suffer from low output powers, thus reducing their effectiveness in a long haul communication system.
The SG-DBR laser is an ideal candidate due to its large tuning range (40 $\mathrm{nm})$, high output power, large Side Mode Suppression Ratio (SMSR) and its ability to be monolithically integrated with other semiconductor devices such as a Semiconductor Optical Amplifier (SOA) or an Electro-Absorption Modulator (EAM) [3]. The SG-DBR is comprised of a front mirror, back mirror, gain and phase section. The front and back mirrors are sampled at different periods such that only one of their reflection peaks can coincide within the range of the gain spectrum at a given set of currents. These peaks are spaced apart in wavelength, at a period inversely proportional to the period of the sampling. Through this technique, the desired ITU (International Telecommunication Union) channel can be selected by tuning the two mirrors, with the closest reflection peak of each mirror aligned at the desired channel [4].

When the tunable laser is set to switch from one ITU channel to another, it generates several spurious components, which interfere with other channels when the tunable laser is employed in a DWDM system. This can lead to unacceptable penalties in system performance, as shown previously in $[5,6]$. In order to eliminate the unwanted spurious components the tunable laser module employs a monolithically integrated SOA at the output. This device remains unbiased for $60 \mathrm{~ns}$ after the beginning of the switch, attenuating the output of the tunable laser during the initial phase of the wavelength tuning. This essentially blanks the spurious components for a significant time during the switch from one ITU frequency to another, thus circumventing any cross channel interference.

Another important aspect of the tunable lasers performance, when switching between wavelengths, is the wavelength stability of the device. As the laser tunes into its desired wavelength, there is a settling drift before the channel finally stabilizes. This drift can have a large impact on the performance of a dense wavelength division multiplexed system (causing adjacent channel interference) and therefore must be fully characterized, in terms of both the magnitude of the drift and the time it takes to settle to the destination wavelength.

Thus far, external modulation has been the most popular technique used with tunable lasers, allowing 
high-speed intensity modulation of the transmitter. Although external modulators provide high-speed, stable data modulation, they introduce loss on the transmitted signal due to their large coupling and insertion losses. The extra optical component also adds to the cost and complexity of the transmitter [7]. On the other hand, direct modulation is one of the simplest and most efficient techniques that could be used to modulate a lightwave signal. Additional benefits associated with direct modulation also include the reduced cost and a smaller transmitter footprint. It is therefore viable to investigate the performance of a directly modulated tunable laser to verify its usefulness in a DWDM system. When a static channel generated from the tunable laser is directly modulated; the direct modulation itself will cause a time dependant frequency drift. This drift can cause poor system performance, due to cross channel interference, thus making it imperative to characterize the magnitude and settling time of the drift in order to optimize system performance.

This paper is divided into three main sections. Section II focuses on the characterization of the frequency drift due to switching events in the tunable laser. The drift is characterized for both magnitude and the time it takes the channel to settle into its targeted ITU frequency. Section III analyzes the direct modulation induced frequency drift on the tunable laser and its effect on the performance of a wavelength division multiplexed system. The latter is achieved by passing the modulated channel through an Optical Band-Pass Filter (OBPF) to mimic a wavelength division multiplexed system receiver. Finally, section IV presents a linewidth characterization, which is vital, if the TL is to be used as a source in systems employing advanced modulation formats. A tuning map as a function of the front and back gratings is presented, which allows the choice of an optimum operating point for such systems (high SMSR and narrow linewidth).

\section{FREQUenCy DRIFT DUE to WAVELENGTH SWITCHING EVENTS}

As mentioned previously, a vital aspect of tunable laser performance is wavelength stability after the laser switching event. Here we measure the instantaneous frequency drift of the tunable laser by using an optical filtering technique. The peak of the filter passband is initially set to the target wavelength, after which it is detuned by $\sim 0.1 \mathrm{~nm}$ to create a sloped frequency discriminator. This process enables us to relate the signal's power variation to the corresponding frequency variation, through the filter reflection profile, thus providing an accurate frequency drift as a function of time.

\section{A. Experimental Setup}

The settling drift of the tunable laser after blanking is characterized using the experimental setup depicted in Fig. 1. The tunable laser used for this work is an AltoNet 1200 fast wavelength switched transmitter that allows switching from one channel to any other channel, over the entire C-band wavelength range, in $200 \mathrm{~ns}$. The tunable laser was supplied by Intune Networks and featured a custom made SMA connector to enable direct modulation of the gain section of the device. The drift of the TL after blanking was measured using a tunable fiber Bragg grating (FBG) with a $3 \mathrm{~dB}$ bandwidth of $27.5 \mathrm{GHz}$ [8]. Initially, a power reference measurement (shown be the dotted line in Fig. 1) is recorded, where the output of the TL is detected by a $50 \mathrm{GHz}$ photodiode in conjunction with a $50 \mathrm{GHz}$ sampling oscilloscope. Subsequently, the switching signal is passed through the OBPF to filter out the targeted ITU channel.

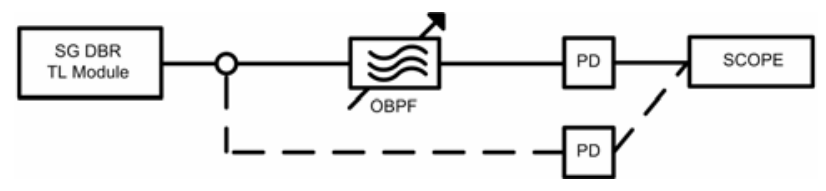

Figure 1. Frequency drift measurement set-up.
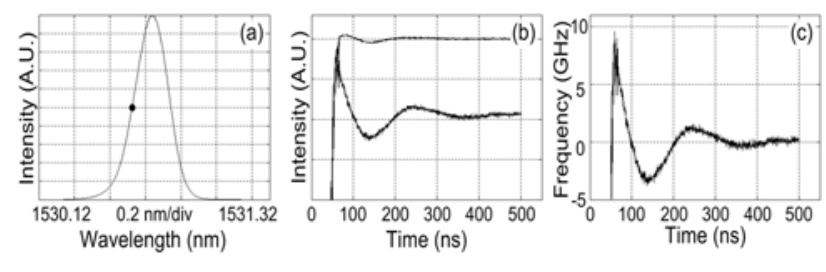

Figure 2. (a) Fiber Bragg grating reflection profile, (b) turn on power transient of laser and signal power with filter detuned by $0.1 \mathrm{~nm}$ and (c) frequency drift measurement

\section{B. Results and Discussion}

As illustrated by Fig. 2a, the optical band-pass filter is de-tuned by $0.1 \mathrm{~nm}$ (black dot) to create a sloped frequency discriminator. When the light from the tunable laser is passed though the discriminator, the frequency fluctuations manifest as intensity variations as seen in Fig. 2b. The turn on power transient of the laser (thin line in Fig. 2b) is subtracted from the filtered signal to ensure that any variation of the received power is only due to the settling drift of the tunable laser. From the filter profile, we know the wavelength or frequency at the steady state level of the measured trace. Thus, through the filter reflection profile, we can relate the power variation to the corresponding frequency variation, therefore providing us with an accurate measurement of the drift as a function of time. The drift measurement was performed for a number of channel transitions. We present the result for the transition between channel $13(192.1 \mathrm{THz})$ and channel $89(195.9 \mathrm{THz})$. This was found to have one of the larger drifts of approximately $10 \mathrm{GHz}$ in magnitude as illustrated in Fig. 2c.

As the tunable laser emerges from blanking it is approximately $10 \mathrm{GHz}$ from its target frequency. The wavelength locker can be seen to turn on 30 ns after blanking. The locker causes a fast fluctuation in frequency for approximately $15 \mathrm{~ns}$, after which the settling drift is characterized by a damped oscillation. The channel settles into its ITU frequency in about 400 ns. In a DWDM system, where channel spacing is as low as 12.5 or $50 \mathrm{GHz}$, the magnitude of this frequency drift would lead to poor system performance due to cross 
channel interference. Hence, the impact of such a drift on the performance of a DWDM system is examined in the following section.

\section{Performance Degradation due to Switching Drift}

The set-up shown in Fig. 3 is employed to investigate the effect of the measured wavelength drift in a dense wavelength division multiplexed network. A twotransmitter DWDM system with channel spacing of 12.5 $\mathrm{GHz}$ is investigated. The tunable is set to switch between channel 42 and 52. A fixed laser is used as a second transmitter operating $12.5 \mathrm{GHz}$ away from the tunable laser target channel at $1544.824 \mathrm{~nm}$. Each channel is externally modulated with a 2.5 Gbit/s Non-Return-toZero (NRZ) pseudo-random bit sequence with a pattern length of $2^{7}-1$.

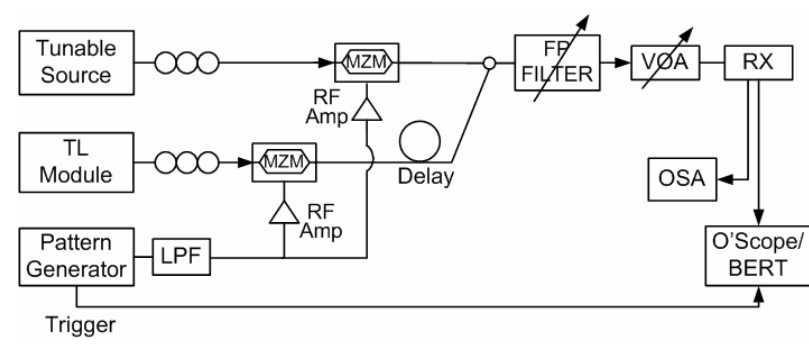

Figure 3. Set-up to investigate the effect of the measured wavelength drift in a DWDM network



Figure 4. BER measurements for $12.5 \mathrm{GHz}$ channel spacing with default blanking time $(\boldsymbol{\Delta})$ and of $12.5 \mathrm{GHz}$ channel spacing using and extended blanking time of 200 ns (०)

The same data is used for both lasers, therefore it is necessary to de-correlate the information carried in each channel. This is achieved by passing one of the channels through $3 \mathrm{~m}$ of fiber. The two channels are then combined together and the fixed channel is filtered out using a Fabry-Perot (FP) tunable filter with a $3 \mathrm{~dB}$ bandwidth of $6 \mathrm{GHz}$. The demultiplexed channel then passes through a Variable Optical Attenuator (VOA) before entering the receiver stage which consists of an Erbium Doped Fibre Amplifier (EDFA), an optical filter, a photodiode and an electrical amplifier. The signal spectrum, eye diagram and the BER of the detected channel are also examined.

The BER of the filtered channel is measured as a function of the received optical power for various tunable laser configurations - when the interfering tunable laser was (a) set to channel 42 (> $500 \mathrm{GHz}$ away from filtered channel), (b) set to channel 52 (12.5 GHz from filtered channel) and (c) switching from channel 42 to channel 52. The tunable laser is set to switch at a rate of $5 \mathrm{kHz}$. There is a minimal power penalty $(<0.1 \mathrm{~dB})$ when the tunable laser operates in a static mode at channel 52 (i.e. $12.5 \mathrm{GHz}$ from the fixed channel) compared to when the tunable laser is static at channel 42 . When the tunable laser switches between channels 42 and 52 the wavelength drift, after blanking, degrades the system performance by introducing an error floor at $10^{-7}$. This bit error rate is the average number of errors on the fixed channel over (a) the time at the beginning of the data transmission when the tunable laser is settling into channel 52, (b) the time when the tunable laser is settled into channel 52, (c) the time when the tunable laser is at channel 42 and (d) the time when the tunable laser is blanked. The switching tunable laser causes a burst of errors only at the beginning of the data stream, as it emerges from blanking and begins to settle into channel 52. At this time the drift is large enough such that it enters the filter passband of the fixed channel. As the tunable laser approaches its target wavelength the errors reduce [9], eventually giving no errors when the tunable laser is within $\sim 3 \mathrm{GHz}$ of its target wavelength. Thus if the tunable laser can be locked with less drift then the interference can be reduced.

The laser blanking time after the wavelength transition is initiated is subsequently increased from the default value of $60 \mathrm{~ns}$ to $200 \mathrm{~ns}$. This is done in anticipation that the laser wavelength will be closer to the target wavelength once the blanking time ends, however this will also result in a corresponding increase in the effective tunable laser switching time, thereby reducing network throughput. The improvement in system performance is also illustrated in Fig. 4. With the extended blanking time the system performs with a residual power penalty of $\sim 1.1 \mathrm{~dB}$ (relative to the case when the tunable laser is static), at a reference bit error rate of $10^{-9}$, due to interference from the adjacent tunable laser channel. This power penalty could not be reduced by further increasing the blanking time as we believe it is primarily due to the frequency drift induced by the locking mechanism.

\section{FREQUENCY DRIFT DUE TO DiRECT ModUlation}

As mentioned earlier, direct modulation is the simplest and most cost efficient method to modulate the lightwave signal generated from a tunable laser. An inherent problem of direct modulation is a shift in the operating frequency due to a large variation of the carrier density upon the application of the modulating current. This shift in frequency will cause adjacent channel interference, thus decreasing the system performance Therefore, it is of vital importance to investigate the magnitude of this drift and the effect it may have if utilized in a dense wavelength division mulitplexed system. 


\section{A. Experimental Setup}

The set-up used to characterize the frequency drift is similar to that employed to characterize the settling drift due to a switching event, with the addition of a Pulse Pattern Generator (PPG), as seen in Fig. 3a. The tunable laser is operated in static mode and the emission frequency is set to channel 38 (193.35 THz). The gain section (via an SMA input) of the tunable laser is then directly modulated with a series of different pattern sequences. As with the switching drift measurement, the optical band pass filter is de-tuned by $0.1 \mathrm{~nm}$ to create a sloped frequency discriminator [10]. The frequency discriminator essentially assists in characterising the offset in frequency of the tunable laser, from the set ITU frequency. By detuning the filter, the set ITU frequency is made to lie on a portion with a higher rejection (lower power) of the filter transfer characteristic. On the application of the modulating signal the frequency of the tunable laser is offset towards a lower rejection side of the filter transmission resulting in a higher amount of optical power being detected. Subsequently, as the inbuilt wavelength locker drags the output signal back to its target frequency, a smaller output power from the filter is recorded.
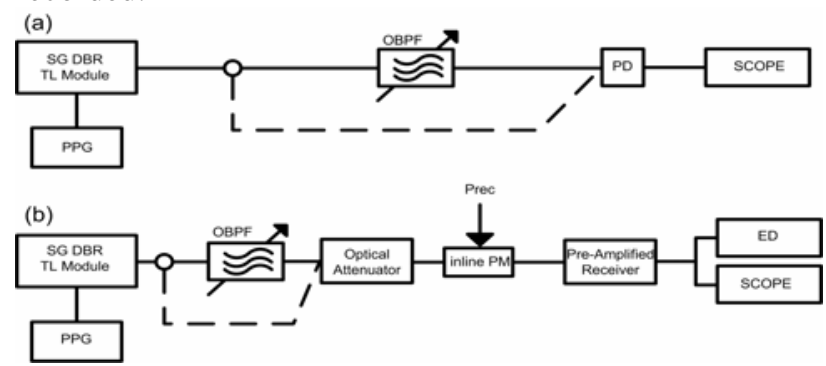

Figure 5. Set-up to measure (a) frequency drift due to direct modulation and (b) effect of the drift when filtered

The power measurement is subsequently subtracted from the filtered signal to ensure that any variation in received power is due to the frequency drift of the tunable laser (and not due to the intensity modulation). To accurately measure the magnitude and settling time of the drift, a programmable bit sequence of thirty ones and thirty zeros is applied to the tunable laser. An average shift of $7 \mathrm{GHz}$ (towards lower frequencies) from the specified frequency (unmodulated emission frequency of tunable laser at channel 38) is noticed as the modulation is applied to the laser.

\section{B. Results and Discussion}

Fig. 4 illustrates the frequency drift as a function of time. The magnitude of the drift is approximately $12 \mathrm{GHz}$ which could have a large impact on an adjacent channel if employed in an ultra dense wavelength division multiplexed system. The offset frequency is then pulled back to its targeted frequency by the wavelength locker incorporated in the tunable laser module. This frequency lies on a lower portion of the filter transfer characteristic, thus exhibiting higher rejection, producing a lower output power. As can be seen from Fig. 4, the output frequency settles at its target wavelength after approximately $18 \mathrm{~ns}$.
To perform a qualitative analysis of the effect the frequency drift will have in a dense wavelength division multiplexed system, Bit Error Rate (BER) measurements are performed. The experimental setup is depicted in Fig. $3 \mathrm{~b}$ and again consisted of a static tunable laser channel $(193.35 \mathrm{THz})$ modulated with a $1 \mathrm{~Gb} / \mathrm{s}$ pseudo random bit sequence of length $2^{7}-1$. The peak to peak voltage of the modulating signal is set at one volt. An optical attenuator and an inline power meter $\left(\mathrm{P}_{\text {rec }}\right)$ are incorporated into the set-up to monitor the varied received power falling on the pre-amplified receiver. The bit error rate measurements are carried out with the aid of an error detector for three different scenarios, namely: no filter (dotted line), with the $27.5 \mathrm{GHz}$ optical band pass filter centered at the targeted ITU channel (193.35 THz) and with the filter centered at the average shifted frequency upon direct modulation (193.343 THz).

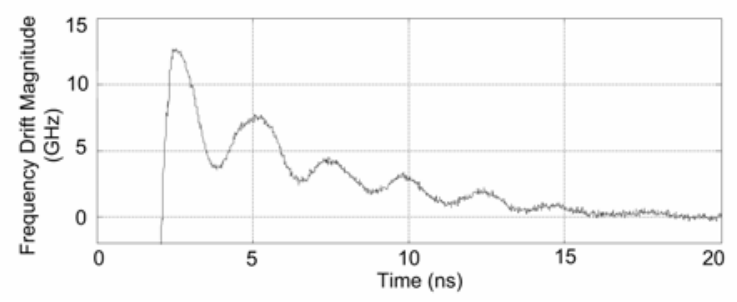

Figure 6. Single channel frequency drift due to direct modulation

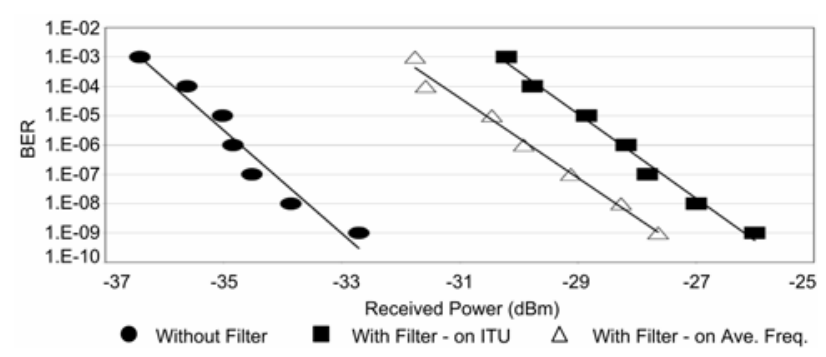

Figure 7. BER versus received power for three scenarios

Fig. 5 illustrates the effect the frequency drift has on the performance of a typical dense wavelength division multiplexed system, by plotting the received power as a function of the bit error rate. The back to back reference curve highlights the systems performance in the case where no filter is used $(\bullet)$. It exhibited a receiver sensitivity of $-33 \mathrm{dBm}$ at an error rate of $10^{-9}$. In contrast, when the modulated signal is filtered with the optical band pass filter centered at the specified frequency of channel 38, the induced frequency deviations caused by the direct modulation result in intensity fluctuations at the output of the filter. These intensity fluctuations are reflected in a worsening system performance shown by the incurred power penalty of $6.7 \mathrm{~dB}$ at a bit error rate of $10^{-9}(\boldsymbol{\square})$. A small improvement in performance (1.64 dB at a BER of $10^{-9}$ ), relative to the latter case, is achieved when the center frequency of the optical band pass filter is tuned to match the average shifted frequency of the tunable laser under modulation $(\Delta)$.

Eye diagrams are also recorded with a $50 \mathrm{GHz}$ sampling oscilloscope for each of the discussed permutations. The eye diagrams further illustrate the bit 
error rate degradation depicted in Fig 5. Fig. 6a shows the received eye when no filter is employed prior to the detected signal. A clear, wide eye opening is displayed, thus supporting the excellent performance with received powers in the order of $-33 \mathrm{dBm}$ at a BER of $10^{-9}$. However, in the case of Fig. 6b, where the filter is centered at the targeted frequency, the large intensity fluctuations caused by the drift in the directly modulated signal results in a partially closed eye, which in turn leads to a power penalty of $6.7 \mathrm{~dB}$. Fig. $6 \mathrm{c}$ represents the received eye when the OBPF is tuned to the average shifted frequency, demonstrating improved system performance in comparison to Fig. $6 \mathrm{~b}(1.64 \mathrm{~dB}$ at a BER of $\left.10^{-9}\right)$.

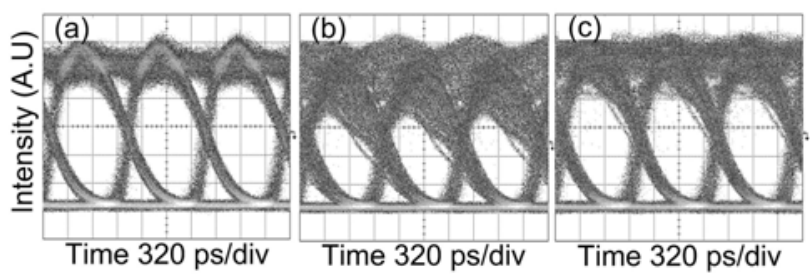

Figure 8. Eye diagrams (a) without filter, (b) with filter centered at targeted ITU frequency and (c) with filter centered at average shifted frequency

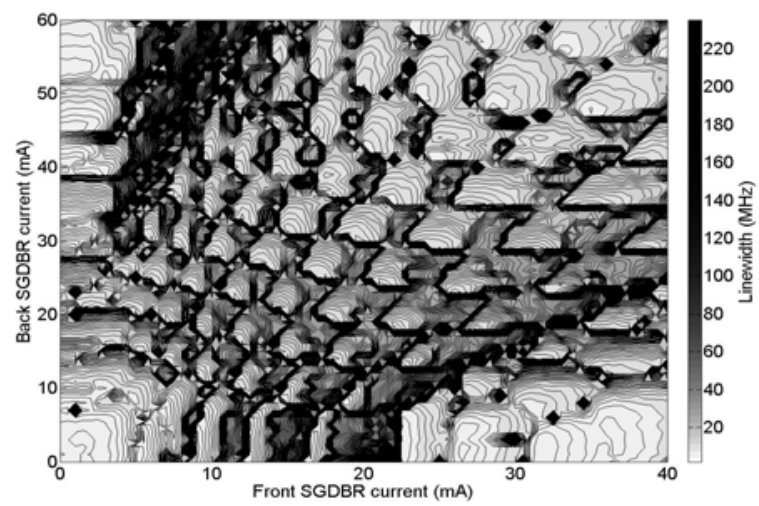

Figure 9. SG DBR Linewidth tuning map as a function of front and back mirror currents

\section{LINEWIDTH CHARACTERIZATION}

In addition to network reconfigurability, tunable lasers are also being investigated as dynamic sources for advanced modulation formats. The introduction of fast reconfigurable optical networks and advanced modulation formats will place additional constraints on the tunable laser transmitters that can be used in such systems. In order to employ an SG-DBR laser as a continuous wave source for advanced modulation formats, it will become imperative to select an optimum operating point to achieve the narrowest possible linewidth to support phase modulated coding schemes. We have employed the selfheterodyne method to measure the linewidth of a Sampled Grating Distributed Bragg Reflector tunable laser. This method was used due to its simple setup, insensitivity to slow wavelength drift and ability to measure narrow linewidths $(5 \mathrm{kHz})$. The linewidth characterization setup is the same as in [11]. The Sampled Grating Distributed Bragg Reflector tunable laser used for this characterization was a commercially available device from Agility Communications and allowed access to all four laser sections.

The linewidth tuning map as a function of the front and back grating currents is illustrated in Fig. 9. The current of the front SG-DBR is scanned from 0 to $40 \mathrm{~mA}$ with a $0.5 \mathrm{~mA}$ step, while the current of the back grating is scanned from 0 to $60 \mathrm{~mA}$ with a $1 \mathrm{~mA}$ step. From Fig. 9 we can see that each mode is surrounded by points with relatively high values of linewidth at the mode boundaries, where frequency jumps occur [9]. Similar side mode suppression ratio and output power tuning maps can also be utilized in conjunction with the linewidth characterization to ensure that acceptable levels of transmitter performance are achieved. From Fig. 9, it is evident that the linewidth alternates significantly from 20 $\mathrm{MHz}$ to as low as $4 \mathrm{MHz}$ within each mode. If these lasers are to be employed in systems that use advanced modulation formats it will become necessary to include the linewidth as an important characteristic when choosing the optimum operating point.

\section{CONCLUSIONS}

We have characterized the drift of a Sampled Grating Distributed Bragg Reflector tunable laser for both, switching events (settling drift), and under the influence of direct modulation (frequency drift). Switching was achieved between two wavelength channels on the ITU grid. The magnitude and the settling time of the drift were characterized, as both factors would have serious implications in a dense wavelength division multiplexed system. A settling drift magnitude of $10 \mathrm{GHz}$ was experienced with a settling time of 400 ns. In a dense wavelength division multiplexed system, where channel spacing is as low as $12.5 \mathrm{GHz}$, the magnitude of this drift could lead to poor system performance due to cross channel interference.

The frequency drift was also characterized when the TL was subjected to direct modulation. The magnitude of the drift has been recorded as $12 \mathrm{GHz}$ with a peak to peak modulation of one volt. In dense wavelength division multiplexed systems this drift may cause performance degradation, again due to cross channel interference and also when passed through an optical filter. Results achieved demonstrate that a power penalty of $6.7 \mathrm{~dB}$ is incurred when using such a filter in comparison to an unfiltered case. A slight improvement in performance (1.64 $\mathrm{dB}$ at $\left.10^{-9}\right)$ is achieved when the filter is tuned to the average shifted frequency of the tunable laser under direct modulation.

The final characterization of the tunable laser involved the measurement of the linewidth as a function of the front and back mirror currents. A linewidth map has been created showing that the purity of the signal generated by tunable laser strongly depends on the value of these currents and can vary (within a mode) from 20 to $4 \mathrm{MHz}$. 


\section{ACKNOWLEDGMENT}

The authors wish to thank Intune Networks Ltd., Ireland for the modifications made to the AltoNet FTL Tx Module. This work was supported in part by a grant from Enterprise Ireland and Science Foundation Ireland.

\section{REFERENCES}

[1] J. E. Simsarian and L. Zhang, "Wavelength locking a fastswitching tunable laser," IEEE Photon. Technol. Lett., vol. 16, no. 7, pp. 1745-1747, Aug. 2004.

[2] L.A. Coldren et al., "Tunable semiconductor lasers: A tutorial,” IEEE/OSA J. Lightwave Technol., vol. 22, no. 1, pp.193-202, Jan. 2004.

[3] L.A. Johansson, J. T. Getty, Y. A. Akulova, G. A. Fish and L. A. Coldren, "Sampled-grating DBR laser-based analog optical transmitters," IEEE J. Lightwave. Technol., vol. 21, no. 12, pp. 2968-2976, Dec. 2003.

[4] J. Klamkin, J.M. Hutchinson, J.T. Getty, L.A. Johansson, E.J. Skogen, L.A. Coldren, "High efficiency widely tunable SG-DBR lasers for improved direct modulation performance," IEEE J. of Selected Topics in Quantum Electronics, vol. 11, no. 5, pp. 931-938, 2005.

[5] E. Connolly, A. Kaszubowska-Anandarajah, P. Perry and L.P. Barry, "Impact of tunable laser wavelength drift in a base-band and sub-carrier multiplexed system," Optics Communications, vol. 281, no. 15-16, pp. 4057-4060, August 2008

[6] E. Connolly, F. Smyth, A. K. Mishra, A. KaszubowskaAnandarajah and L. P. Barry, "Cross channel interference due to wavelength drift of tunable lasers in DWDM networks,” IEEE Photon. Technol. Lett., vol. 19, no. 8, pp. 616-618, April 2007.

[7] M. L. Majewski, J. Barton and L. A. Coldren, "Widely tunable directly modulated sampled-grating DBR lasers," in OFC 2002, paper ThV2, pp 537-538.

[8] V.Polo, A. Ausiro, J. Prat and G. Junyent, "GCSR laser frequency drift compensation using optimized current waveform on one single elctrode," in proc. ICTON, paper, We.A1.5, 2005.

[9] Masahiko Fujiwara, Niloy K. Dutta and Achyut K. Dutta, WDM Technologies: Active Optical Components. Boston: Academic Press, 2002.

[10] J.E. Simsarian and L. Zhang, "Wavelength locking a fastswitching tunable laser," IEEE Photon. Technol. Lett., vol. 16, no. 7, pp. 1745-1747, Jul. 2004.

[11] J. Buus, M.C. Amann, and D. J. Blumethal, Tunable laser diodes and related optical sources. Hoboken, N.J.: Wiley Interscience, pp. 223, 2005

Prince M. Anandarajah received his B.Eng (Electronic Engineering) degree from University of Nigeria, Nsukka (UNN) in 1992. From 1993-1997 he worked as an Instructor / Maintenance Engineer in the Aeronautical Telecommunications department, Nigerian College of Aviation Technology (NCAT). He completed his M. Eng., in 1998, at Dublin City University (DCU), Ireland after which he joined the Radio and Optical Communications Laboratory, at the Research Institute for Networks and Communications Engineering (RINCE) also based in DCU. As a result of the research work carried out, specializing in the generation and optimization of pico-second optical pulses, he obtained his PhD degree (2003). From September 2003 until December 2007, he worked as a postdoctoral fellow with the Radio and Optical Communications laboratory.
Currently, he works as a research officer with the High Speed Devices and Systems centre, within RINCE, as a research officer. His main research interests are in the domain of highspeed pulse generation and characterization, tunable lasers, radio over fibre systems and advanced modulation formats.

Aleksandra Kaszubowska-Anandarajah received her BEng. (Electronic and Telecommunication Engineering) from Poznan University of Technology in 2000. In April 2000 she joined the Radio and Optical Communications Laboratory in Dublin City University, Ireland where she was researching on radio over fiber systems. She was awarded her $\mathrm{PhD}$ degree in November 2004. From 2004 till 2008 she was employed as a Postdoctoral Fellow in the Radio and Optical Communications Laboratory at Dublin City University, Ireland. Here, she was worked on the application of fast tunable lasers in radio over fiber systems and wavelength packet switched networks.

In October 2008 she joined Intune Networks Ltd., as a Senior Optical Engineer focusing on the development of a wavelength switched optical packet ring network.

Robert Maher received his B.Eng. degree in electronic engineering in 2005 and since then he has been working towards his $\mathrm{PhD}$ with the Radio and Optical Communications group at the Research Institute for Networks and Communications Engineering (RINCE), based in Dublin City University. The main topic of his research involves the development and characterisation of wavelength tuneable transmitters for reconfigurable optical networks.

Kai Shi received his BSc and M. Eng. degree in 2006 and 2008 respectively from Huazhong University of Science and Technology, Wuhan, China. Since then he has been working towards his $\mathrm{PhD}$ with the Radio and Optical Communications group at the Research Institute for Networks and Communications Engineering (RINCE), Dublin City University. His main research interests are in the area of tunable lasers and advanced modulation formats.

Liam P. Barry received his BE (Electronic Engineering) and MEngSc (Optical Communications) degrees from University College Dublin in 1991 and 1993 respectively. From February 1993 until January 1996 he was employed as a Research Engineer in the Optical Systems Department of France Telecom's Research Laboratories (CNET) in Lannion, France. During this period his research involved the use of ultra short optical pulses in high capacity optical networks, and as a result of this work he obtained his PhD Degree from the University of Rennes in France. In February 1996 he joined the Applied Optics Centre in Auckland University, New Zealand, as a Research Fellow. His work in New Zealand was mainly concerned with optical pulse generation and measurement, and the use of optical nonlinearities for high speed all-optical switching in fibre networks. In March 1998 he took up a lecturing position in the School of Electronic Engineering at Dublin City University, and established the Radio and Optical Communications Laboratory, which is part of the Research Institute for Networks and Communications Engineering (RINCE). He is currently Associate Professor in the School of Electronic Engineering, Director of RINCE, and a Principal Investigator for Science Foundation Ireland. 